\title{
Primary hyperparathyroidism in young patients in Russia: high frequency of hyperparathyroidism- jaw tumor syndrome
}

\author{
Elizaveta Mamedova1, Natalya Mokrysheva², Evgeny Vasilyev³, Vasily Petrov³, \\ Ekaterina Pigarova', Sergey Kuznetsov, Nikolay Kuznetsov, Liudmila Rozhinskaya', \\ Galina Melnichenko, ${ }^{5,6}$, Ivan Dedov7 and Anatoly Tiulpakov ${ }^{3}$
}

\author{
'Department of Neuroendocrinology and Bone Diseases, Endocrinology Research Center, Moscow, Russian Federation \\ ${ }^{2}$ Department of Parathyroid Diseases, Endocrinology Research Center, Moscow, Russian Federation \\ ${ }^{3}$ Department and Laboratory of Inherited Endocrine Disorders, Endocrinology Research Center, Moscow, \\ Russian Federation \\ ${ }^{4}$ Department of Surgery, Endocrinology Research Center, Moscow, Russian Federation \\ 5.M. Sechenov First Moscow State Medical University, Moscow, Russian Federation \\ ${ }^{6}$ Institute of Clinical Endocrinology, Endocrinology Research Center, Moscow, Russian Federation \\ ${ }^{7}$ Endocrinology Research Center, Moscow, Russian Federation
}

Correspondence should be addressed to E Mamedova Email

elisabetta_2010@mail.ru

\begin{abstract}
Background: Primary hyperparathyroidism (PHPT) is a relatively rare disorder among children, adolescents and young adults. Its development at an early age is suspicious for hereditary causes, though the need for routine genetic testing remains controversial. Objective: To identify and describe hereditary forms of PHPT in patients with manifestation of the disease under 40 years of age.

Design: We enrolled 65 patients with PHPT diagnosed before 40 years of age. Ten of them had MEN1 mutation, and PHPT in them was the first manifestation of multiple endocrine neoplasia type 1 syndrome.

Methods: The other fifty-five patients underwent next-generation sequencing (NGS) of a custom-designed panel of genes, associated with PHPT (MEN1, CASR, CDC73, CDKN1A, $C D K N 1 B, C D K N 1 C, C D K N 2 A, C D K N 2 C, C D K N 2 D)$. In cases suspicious for gross $C D C 73$ deletions multiplex ligation-dependent probe amplification was performed. Results: NGS revealed six pathogenic or likely pathogenic germline sequence variants: four in CDC73 c.271C>T (p.Arg91*), c.496C>T (p.Gln166*), c.685A>T (p.Arg229*) and c.787C > T (p.Arg263Cys); one in CASR c.3145G>T (p.Glu1049*) and one in MEN1 c.784-9G>A. In two patients, MLPA confirmed gross CDC73 deletions. In total, 44 sporadic and 21 hereditary PHPT cases were identified. Parathyroid carcinomas and atypical parathyroid adenomas were present in $8 / 65$ of young patients, in whom CDC73 mutations were found in $5 / 8$.

Conclusions: Hereditary forms of PHPT can be identified in up to $1 / 3$ of young patients with manifestation of the disease at $<40$ years of age. Parathyroid carcinomas or atypical parathyroid adenomas in young patients are frequently associated with $C D C 73$ mutations.
\end{abstract}

Endocrine Connections (2017) 6, 557-565

\footnotetext{
Key Words

- primary hyperparathyroidism

- hyperparathyroidism-jaw tumor syndrome

- multiple endocrine neoplasia 1

- familial isolated hyperparathyroidism

- CDC73
}

http://www.endocrineconnections.org DOI: 10.1530/EC-17-0126 (c) 2017 The authors Published by Bioscientifica Ltd

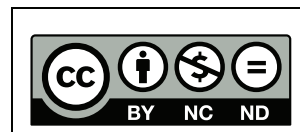

This work is licensed under a Creative Commons Attribution-NonCommercial-NoDerivs 4.0 International License. 


\section{Introduction}

Primary hyperparathyroidism (PHPT) is a common endocrine disorder, predominantly in postmenopausal women (1). Nevertheless, PHPT can occur at any age, and its incidence increases steadily after age 25 years in both sexes (2). The majority of studies on special features of PHPT in young patients demonstrate that in comparison with elderly patients the former have greater morbidity (i.e. symptomatic hypercalcemia, nephrolithiasis, severe bone involvement and nonspecific symptoms) (reviewed in 3).

To date, the following familial syndromes associated with PHPT are known: multiple endocrine neoplasia type 1 (MEN1), type 2A (MEN2A), type 4 (MEN4), hyperparathyroidism-jaw tumor syndrome (HPT-JT), familial hypocalciuric hypercalcemia (FHH) (types 1-3), neonatal severe hyperparathyroidism (NSHPT) and familial isolated hyperparathyroidism (FIHP) (4). The abovementioned syndromes are caused by heterozygous germline mutations in MEN1 (MEN1), RET (MEN2A), CDKN1B (MEN4), CDC73 (formerly HRPT2) (HPT-JT), CASR (heterozygous in FHH type 1 and homozygous in NSHPT), GNA11 (FHH type 2), AP2S1 (FHH type 3). The genetic causes of FIHP in the majority of cases remain unknown, though in some cases germline mutations in MEN1, CDC73 and CASR are identified, indicating that FIHP could be a 'mild' variant of MEN1 and HPT-JT (5).

PHPT as a component of some familial syndromes has its specific features. Thus, PHPT in MEN1 occurs in $75-95 \%$ of patients, it is typically the first manifestation of the disease, the onset is usually between ages 20 and 25 years, and it is caused by multiple parathyroid hyperplasia or adenomas (6). PHPT in HPT-JT has been observed in up to $95 \%$ of affected individuals, with the typical onset in early adulthood, but it can occur at any age, and in about $15 \%$ of cases is caused by parathyroid carcinoma $(4,7)$. PHPT is rarely if ever the first manifestation of MEN2A (5). The typical features of PHPT in MEN4 have not been sufficiently described due to the rarity of the disease (4). FHH is genetically heterogeneous, but FHH type 1 caused by heterozygous mutations in CASR gene is the most common form (8). FHH is usually asymptomatic, and rare complications observed in $\mathrm{FHH}$ consist of clinically silent chondrocalcinosis, premature vascular calcification, pancreatitis and gallstones $(4,8)$. FIHP can result from either the incomplete expression of known hereditary syndromes (MEN1, HPT-JT, FHH) or from unknown genetic causes (5).
PHPT development at an early age is suspicious for hereditary causes, but whether routine mutational analysis among young patients with PHPT, for example for MEN1 mutations, is needed and under what age it is advisable remains controversial (9). There are two studies evaluating the necessity of routine MEN1 sequencing among patients with PHPT under 40 years of age, which in total showed that germline MEN1 mutations had been identified only in three out of 36 patients (8.3\%) with clinically sporadic PHPT $(10,11)$. Another study by Starker and colleagues showed that germline mutations are frequently found among young patients with clinically sporadic PHPT: 4 MEN1, 3 CASR and 1 CDC73 among 86 patients $\leq 45$ years of age $(9.3 \%)(12)$. When these clinically sporadic cases were summarized with PHPT cases diagnosed in the clinical routine, the frequency of germline mutations raised to $23.5 \%(24 / 102,15$ MEN1, 4 RET, 3 CASR, 2 CDC73/HRPT2), which allowed the authors to conclude that the use of genetic testing may be advocated for young PHPT patients even without suspicious family history (12).

Thus, the objective of our study was to identify and describe hereditary forms of PHPT as the first manifestation in patients with the onset of the disease under 40 years of age.

\section{Subjects and methods}

\section{Patients}

The study was approved by the Local Ethics Committee of Endocrinology Research Center (protocol \#8 of 12.11.2013). We enrolled 65 patients ( 47 females and 18 males) with the diagnosis of PHPT, made according to the usual criteria (13), manifested before 40 years of age. Consent has been obtained from each patient after full explanation of the purpose and nature of all procedures used. Median age of manifestation was 24 years, (20; 30), (10; 39). Among them, 44 had clinically sporadic PHPT, ten patients had PHPT as the first manifestation of MEN1 with identified MEN1 mutations (one with FIHP), four patients were from families with FIHP who have not undergone genetic testing and seven patients were suspicious for positive family history (based on the presence of nephrolithiasis, ulcer disease, bone pathology etc. in the first-degree or second-degree relatives). http://www.endocrineconnections.org DOI: 10.1530/EC-17-0126
() 2017 The authors Published by Bioscientifica Ltd
This work is licensed under a Creative Commons Attribution-NonCommercial-NoDerivs 4.0 International License. 


\section{DNA extraction}

Genomic DNA was extracted from peripheral leucocytes using PureLink Genomic DNA Mini Kit according to the manufacturer's protocol (Thermo Fisher Scientific).

\section{Sanger sequencing}

In ten patients, the presence of MEN1 mutations was known before the beginning of the study. In them, the coding exons and exon-intron boundaries of MEN1 had been amplified by PCR and the amplification products underwent Sanger sequencing using ABI 3130 Genetic Analyzer (Applied Biosystems). For PCR, the following primers were used: 1F, 5'-GGG GCG GGT GGA ACC TTA G-3'; 2F, 5'-TTG GGT CAC AGG CTT GGA AAG-3'; 3R, ACA GGG ACC ACC CAC CAT GTG-3'; 8F, 5'-AAG AAT GTT CCC AAC CTA TGC-3'; 9R, 5'-GCA GAA CAT GGG CTC AGA GTT G-3'.

\section{Next-generation sequencing (NGS)}

NGS was performed in 55 patients. A custom-designed panel included genes associated or presumably associated with familial forms of PHPT, except MEN2A and FHH types 2 and 3: MEN1, CASR, CDC73, CDKN1A, CDKN1B, $C D K N 1 C, C D K N 2 A, C D K N 2 C$ and CDKN2D. Primers for multiplex amplification of the abovementioned genes were designed using Ion AmpliSeq Designer (https://www.ampliseq.com). NGS was carried out on semiconductor sequencer Ion Torrent Personal Genome Machine (Thermo Fisher Scientific) according to the manufacturer's protocol. Raw data were analyzed using Torrent Suite (Thermo Fisher Scientific) and alignment of NGS reads was to the reference genome sequence GRCh37/hg19 (http://genome-euro.ucsc. edu/cgi-bin/hgGateway). Reference sequences for the abovementioned genes were as follows: NM_130799.2, NM_000388.3, NM_024529.4, NM_078467.2, NM_004064.4, NM_000076.2, NM_000077.4, NM_001262.2, NM_001800, respectively. ANNOVAR free software (http://annovar.openbioinformatics.org) was used for variant annotation (14). ExomeDepth free software was used for CNV calling (15). Allele frequencies of identified variants were assessed using Exome Aggregation Consortium (ExAC) database (http://exac.broadinstitute.org).

\section{Multiplex ligation-dependent probe amplification (MLPA)}

MLPA was performed in two patients in whom gross CDC73 deletions were suspected according to the ExomeDepth results. MLPA was carried out using SALSA MLPA probemix P466-A1 CDC73 (Lot A1-0415) (MRCHolland, Amsterdam, the Netherlands) and SALSA MLPA EK1-FAM (MRC-Holland); capillary electrophoresis was performed on ABI 3500 Genetic Analyser (Applied Biosystems) with $50 \mathrm{~cm}$ capillary, GeneScan 600 LIZ Size Standard (Applied Biosystems) and POP-7 polymer according to the manufacturer's protocol. MLPA results were analyzed using Coffalyser.Net (MRC-Holland) (https://coffalyser.wordpress.com).

\section{Statistical analysis}

The results are reported as median, 1st and 3rd quartiles (Q1; Q3) and minimum and maximum values (min; max).

\section{Results}

\section{Mutational analysis}

The results of MEN1 Sanger sequencing in ten patients with MEN1 mutations are shown in Table 1. There were two nonsense mutations, two frameshift mutations, two splicing mutations and four missense mutations.

The results of NGS are shown in Table 2. There were three nonsense and one missense CDC73 mutations, three of which were not previously described. One nonsense heterozygous CASR mutation affecting the C-terminal tail of the receptor and one splicing MEN1 mutation were also identified. Allele frequencies of all the identified mutations were not found in ExAC. No pathogenic or likely pathogenic variants were identified in CDKIs genes.

Besides, ExomeDepth showed a reliable $(\mathrm{BF}=33.4)$ decrease to 0.572 in the observed relative to expected read depth for CDC73 22 consecutive amplicons (exons 1-17, the whole gene) in one patient and a reliable $(\mathrm{BF}=34.1)$ decrease to 0.602 in the observed relative to expected read depth for CDC73 14 consecutive amplicons (exons 1-10) in another patient. This could indicate the existence of large heterozygous deletions of these loci respectively. These two patients underwent MLPA analysis, which confirmed the deletion of the whole gene and four 
Table 1 The results of MEN1 Sanger sequencing.

\begin{tabular}{|c|c|c|c|c|c|c|c|}
\hline Patient \# & Sex & Gene & Exon & CDNA & Amino acid change & $\begin{array}{l}\text { Type of } \\
\text { mutation }\end{array}$ & $\begin{array}{l}\text { Description in } \\
\text { the literature }\end{array}$ \\
\hline 1 & Female & MEN1 & 2 & c.247_250delCTGT & p.Thr85SerfsTer33 & Frameshift & Not described \\
\hline 2 & Female & MEN1 & 3 & c.628_631delACAG & p.Ser210fsTer222 & Frameshift & (16) \\
\hline 3 & Female & MEN1 & Intron 3 & c. $654+1 G>A$ & - & Splicing & (17) \\
\hline 4 & Female & MEN1 & Intron 3 & c. $654+1 \mathrm{G}>\mathrm{A}$ & - & Splicing & $(17)$ \\
\hline 5 & Female & MEN1 & 4 & c.658T >C & p.Trp220Arg & Missense & Not described \\
\hline 6 & Female & MEN1 & 4 & c.719_720TG >AA & p.Val240Glu & Missense & Not described \\
\hline 7 & Female & MEN1 & 4 & c. $728 \mathrm{~T}>\mathrm{A}$ & p.lle243Asn & Missense & Not described \\
\hline 8 & Female & MEN1 & 7 & c. $923 C>G$ & p.Ser308* & Nonsense & (18) \\
\hline 9 & Female & MEN1 & 9 & c. $1243 C>T$ & p.Arg415* & Nonsense & (19) \\
\hline 10 & Male & MEN1 & 9 & c. $1252 \mathrm{G}>\mathrm{A}$ & p.Asp418Asn & Missense & (20) \\
\hline
\end{tabular}

additional genes (TROVE2, GLRX2, B3ALT2, LINK0103) in one case and a large deletion of exons 1-10 in another case respectively (Fig. 1).

\section{Clinical data}

The genetic analysis allowed us to divide the whole group of 65 patients into several subgroups: (1) sporadic PHPT (i.e. without identified germline mutations or relatives with PHPT) (44/65); (2) HPT-JT (6/65), including one patient with FIHP; (3) MEN1 (11/65), including one patient with FIHP; (4) one patient with CASR mutation $(1 / 65)$ and (5) FIHP without identified mutations (3/65). We focus on each group below.

Among sporadic PHPT cases, there were 30 females and 14 males $(2: 1)$. The mean age at PHPT diagnosis was 25 years $(21 ; 30),(10 ; 39)$. There were $16 / 44$ of mild forms and 28/44 of manifest forms. Eleven patients had severe osteitis fibrosa cystica (two with parathyroid carcinoma, one with atypical parathyroid adenoma and eight with solitary parathyroid adenoma). Forty-three patients underwent surgical treatment of PHPT. The majority of cases had histological diagnosis of a solitary parathyroid adenoma (35/43). The rest had: solitary parathyroid hyperplasia (3/43), one patient had two parathyroid adenomas (1/43), one patient had one parathyroid adenoma and one parathyroid hyperplasia (1/43), two male patients had parathyroid carcinoma (2/43) and one female patient had atypical parathyroid adenoma (1/43). Thus, 3/43 of patients with sporadic PHPT under 40 had either parathyroid carcinoma or atypical parathyroid adenoma. All patients achieved remission after surgical treatment. It is noteworthy that five patients with sporadic PHPT had suspicious positive family history (in two patients, nephrolithiasis in first-degree relatives; in two patients, nephrolithiasis in second-degree relatives; in one patient, ulcer disease in a first-degree relative).

Clinical characteristics of patients with genetically confirmed HPT-JT are summarized in Table 3 . The majority of patients $(5 / 6)$ either with nonsense CDC73 mutations or gross CDC73 deletions had parathyroid carcinomas and severe PHPT. Only one female patient with mild PHPT due to a solitary parathyroid hyperplasia had a missense CDC73 mutation. Patient \#17 aged 13 years at the time of PHPT manifestation who had parathyroid carcinoma and a gross deletion of the whole CDC73 gene was from a FIHP family - her mother also had PHPT caused by a solitary parathyroid adenoma. Two other patients were suspicious for positive family history (patient \#11 had a first-degree relative with an end-stage renal disease as a consequence of polycystic kidney disease, patient \#18 had a first-degree relative presumably with Calvé's disease). All patients achieved remission after parathyroidectomy except patient \#1, in whom PHPT was diagnosed with multiple lung metastases of parathyroid carcinoma (this clinical case was thoroughly described previously (24)).

Table 2 The results of NGS.

\begin{tabular}{llll} 
Patient \# & Sex & Gene \\
\cline { 1 - 1 } 11 & female & CDC73 \\
12 & female & CDC73 \\
13 & female & CDC73 \\
14 & female & CDC73 \\
15 & female & CASR \\
16 & male & MEN1 \\
\hline
\end{tabular}

\begin{tabular}{|c|c|c|}
\hline GRCh37/hg19 location & Exon & CDNA \\
\hline chr1:193099337C>T & 3 & c. $271 \mathrm{C}>\mathrm{T}$ \\
\hline chr1:193107287C>T & 6 & c. $496 C>T$ \\
\hline chr1:193111152A>T & 7 & c. $685 \mathrm{~A}>\mathrm{T}$ \\
\hline chr1:193117054C>T & 8 & c. $787 \mathrm{C}>\mathrm{T}$ \\
\hline chr3:122003946G>T & 7 & c.3145G >T \\
\hline chr11:64577619C>T & Intron 4 & c.784-9G >A \\
\hline
\end{tabular}

\begin{tabular}{l} 
Amino acid \\
change \\
\hline p.Arg91* \\
p.Gln166* \\
p.Arg229* \\
p.Arg263Cys \\
p.Glu1049* \\
-
\end{tabular}

\begin{tabular}{l}
$\begin{array}{l}\text { Type of } \\
\text { mutation }\end{array}$ \\
\hline Nonsense \\
Nonsense \\
Nonsense \\
Missense \\
Nonsense \\
Splicing
\end{tabular}

\begin{tabular}{l}
$\begin{array}{l}\text { Description in } \\
\text { the literature }\end{array}$ \\
\hline$(12,21)$ \\
Not described \\
Not described \\
Not described \\
Not described \\
$(22,23)$
\end{tabular}

\begin{tabular}{c}
$\begin{array}{c}\text { Read } \\
\text { depth }\end{array}$ \\
\hline $267 x$ \\
$246 x$ \\
$267 x$ \\
$313 x$ \\
$383 x$ \\
$297 x$
\end{tabular}



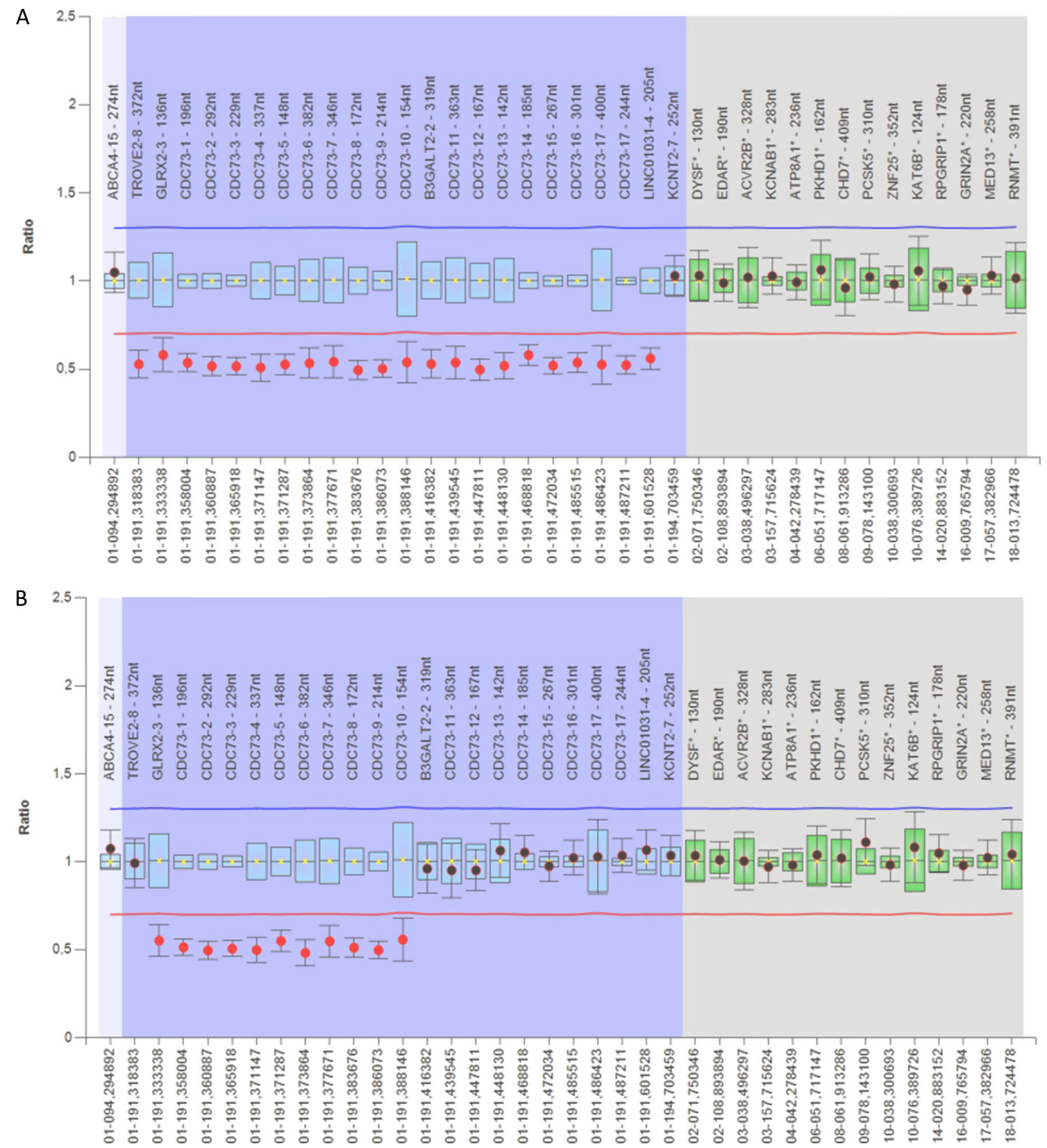

\section{Figure 1}

Results of MLPA analysis show (A) a gross deletion of the whole CDC73 gene in a female with parathyroid carcinoma from FIHP family and (B) a deletion of 1-10 exons of $C D C 73$ gene in a male with atypical parathyroid adenoma.

None of the patients with HPT-JT in our study had jaw, kidney or uterine tumors.

The total number of MEN1 patients with PHPT as the first manifestation of the disease was 11 (10 identified previously by Sanger sequencing and one by NGS). There were nine females and two males $(4.5: 1)$. Median age at the time of PHPT manifestation was 26 years $(18 ; 31),(14,38)$. In six cases (6/11), mild PHPT was present, five patients (5/11) had manifest forms, four of whom had recurrent nephrolithiasis and one patient had osteitis fibrosa cystica. Family history was suspicious in the majority of cases (10/11): one patient was from a FIHP family; two patients had clinical MEN1 diagnosis in first-degree relatives; one patient had genetically confirmed MEN1 in a first-degree relative; one patient had first-degree relatives with hypercalcemia; the remaining four patients had first-degree relatives with either recurrent ulcer disease or tumors of the brain/pancreas. MEN1 group was clinically 
Table 3 Clinical characteristics of patients with HPT-JT.

\begin{tabular}{|c|c|c|c|}
\hline Patient \# & \#11 & $\# 12$ & \#13 \\
\hline CDC73 mutation & p.Arg91* & p.Gln166* & p.Arg229* \\
\hline $\begin{array}{l}\text { Age at } \\
\text { manifestation, years }\end{array}$ & 24 & 18 & 22 \\
\hline PTH, pg/ml & 558.8 & 2440 & 1441 \\
\hline $\begin{array}{l}\text { Serum total calcium, } \\
\mathrm{mmol} / \mathrm{l}\end{array}$ & 3.36 & 4.19 & 3.9 \\
\hline $\begin{array}{l}\text { Serum ionized } \\
\text { calcium, mmol/l }\end{array}$ & 1.56 & N/A & 1.84 \\
\hline PHPT form & Manifest & Manifest & Manifest \\
\hline Histological diagnosis & $\begin{array}{r}\text { Parathyroid } \\
\text { carcinoma }\end{array}$ & $\begin{array}{c}\text { Parathyroid } \\
\text { carcinoma } \\
\text { with lung } \\
\text { metastases }\end{array}$ & $\begin{array}{r}\text { Parathyroid } \\
\text { carcinoma }\end{array}$ \\
\hline Family history & $\begin{array}{l}\text { Polycystic } \\
\text { kidney disease } \\
\text { in mother }\end{array}$ & Unremarkable & Unremarkable \\
\hline
\end{tabular}

$\frac{\# 14}{\text { p.Arg263Cys }}$
30
125.1
2.94
1.24

\begin{tabular}{c}
\hline $\mathbf{\# 1 7}$ * \\
\hline Ex. 1-17 \\
13 \\
1550
\end{tabular}

1.58

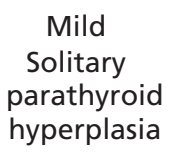
hyperplasia

Unremarkable

\section{Manifest \\ Parathyroid \\ carcinoma}

PHPT in mother (FIHP)

\begin{tabular}{c}
\hline $\mathbf{\# 1 8}$ \\
\hline Ex. 1-10 \\
18 \\
1833 \\
4.49 \\
2.03
\end{tabular}

Manifest

Atypical

parathyroid

adenoma

Calvé's disease

(?) in sister

*, A proband from a FIHP family; N/A, not available; PTH, parathyroid hormone.

heterogeneous, as shown in Table 4. Most patients had recurrence of PHPT after surgical treatment and seven patients had pituitary adenomas, neuroendocrine tumors or other tumors diagnosed after PHPT. Nobody had a histological diagnosis of parathyroid carcinoma or atypical parathyroid adenoma.

One female patient with a nonsense CASR mutation had mild PHPT diagnosed incidentally at 23 years of age. She had serum total calcium $3.01 \mathrm{mmol} / \mathrm{L}$ (2.15-2.55), ionized calcium $1.33 \mathrm{mmol} / \mathrm{L}$ (1.03-1.29), phosphorus $0.81 \mathrm{mmol} / \mathrm{L} \quad(0.74-1.52)$, serum PTH $147.6 \mathrm{pg} / \mathrm{mL}$ (15-65), urinary calcium/creatinine clearance ratio (UCCR) 0.0116. Ultrasound revealed lower left parathyroid tumor. The patient underwent lower left parathyroidectomy with intraoperative lowering of the PTH to $16.2 \mathrm{pg} / \mathrm{mL}$ and postoperative hypocalcemia. Histology revealed a solitary parathyroid adenoma. The patient also had type 1 diabetes mellitus and autoimmune thyroiditis diagnosed at 14, and celiac disease diagnosed at 29. Whether the identified mutation alters calcium-sensing receptor function remains unknown. Its pathogenicity is questionable as FHH is characterized by persistent hypercalcemia after surgical intervention.

Overall, there were five FIHP families in our study, in two of which a genetic cause of the disease was revealed: MEN1 mutation p.Asp418Asn in one family and a gross CDC73 deletion of the whole gene in another. In three families, no pathogenic sequence variants were identified. The latter three probands had PHPT remission after surgical solitary parathyroid adenoma removal 3, 6 and 7 years postoperatively respectively. In two families without identified mutations two members were affected (mother and daughter). In the third family, four members were affected, and it is noteworthy that the proband had one child with bilateral retinoblastoma and another with congenital renal abnormalities.

\section{Discussion}

PHPT is a sporadic disorder in the majority of cases, and only $5-10 \%$ are associated with familial syndromes (1). Among them, MEN1 is the most frequent form of hereditary PHPT, and it comprises $1-18 \%$ of all PHPT cases (6), while HPT-JT, FHH and FIHP occur much rarely (4). For all these syndromes, it is recognized that PHPT typically manifests at a younger age in comparison with sporadic PHPT, thus making young age at diagnosis suspicious for the presence of one of the familial syndromes $(4,9)$.

In our study, sporadic PHPT occurred in approximately $2 / 3$ of patients under 40 years of age. Consistent with other studies (reviewed in 3) our results show that sporadic PHPT in young patients is usually diagnosed as manifest forms (28/44), and the main cause of PHPT in such cases is a solitary parathyroid adenoma (35/43). Nevertheless, parathyroid carcinoma and atypical parathyroid adenoma were present in 3/43 of sporadic PHPT cases in our cohort. It is noteworthy that suspicious positive family history, a histological diagnosis of parathyroid hyperplasia, parathyroid carcinoma or atypical parathyroid adenoma and the presence of several parathyroid lesions were not necessarily associated with hereditary forms of PHPT.

Parathyroid carcinoma occurs extremely rarely in children and adolescents (3). In HPT-JT, parathyroid (c) 2017 The authors Published by Bioscientifica Ltd
This work is licensed under a Creative Commons Attribution-NonCommercial-NoDerivs 4.0 International License. 


$$
\mid
$$

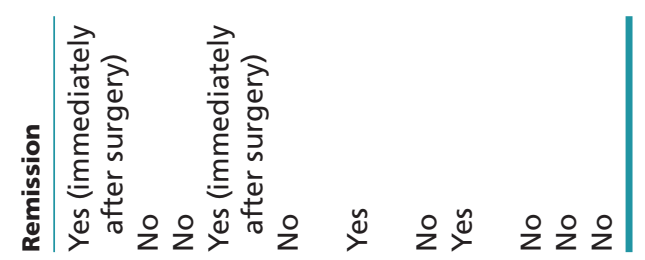$$
1
$$
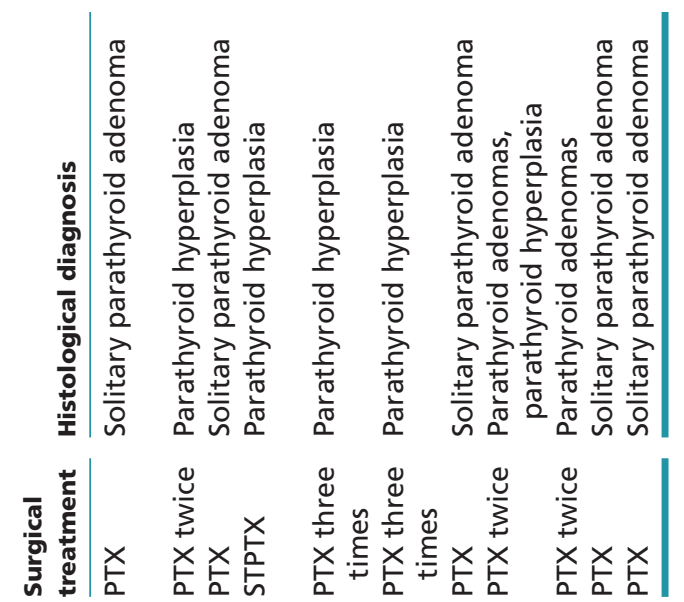

\section{$\stackrel{4}{4}$}

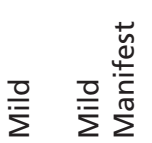

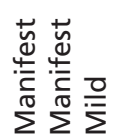

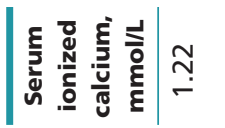

$\checkmark \stackrel{\bar{m}}{-} \overline{\underline{\sigma}}$

$\varangle$

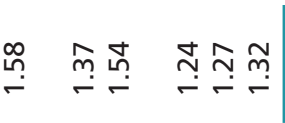

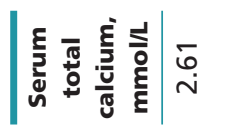

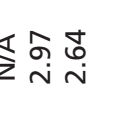

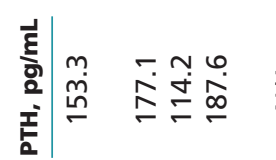

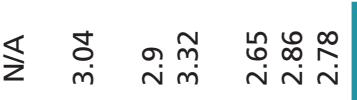

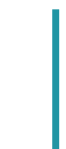

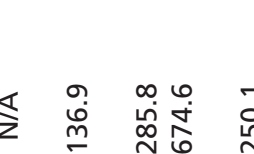

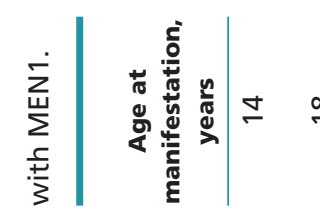

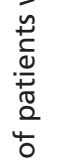

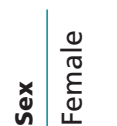

$\stackrel{\sim}{\sim} \stackrel{\sim}{\sim}$

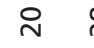

$\infty \bar{m} \bar{m}$

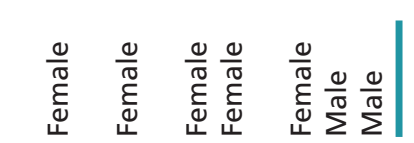

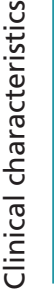

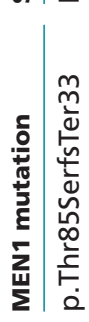

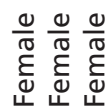

है

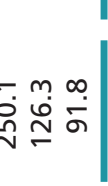
in all patients with parathyroid carcinoma and atypical parathyroid adenoma (both HPT-JT and sporadic cases) as well as in eight sporadic PHPT cases due to a solitary parathyroid adenoma. Thus, an aggressive course of PHPT per se without a histological diagnosis does not always indicate the presence of HPT-JT.

MEN1 is the most frequent form of familial PHPT in our study (11/65). Patients with PHPT as the first manifestation of the syndrome at age under 40 years had an equal proportion of mild and manifest forms. As shown in Table 4, the majority of MEN1 patients in our study had PHPT recurrence after surgical treatment, multiple parathyroid hyperplasia or adenomas and positive family history. All these observations, except the predominance of female patients, are consistent with previous studies $(6,28)$. In our study, only one MEN1 mutation (patient \#16 without positive family history) was identified by NGS, while other mutations in patients were identified

carcinoma is found in $15-20 \%$ of cases (7), while sporadic of cases $(1,25)$. It is generally accepted that all patients with parathyroid carcinomas should undergo Sanger
sequencing of $C D C 73$ gene (7). In our study, there were in total eight cases of parathyroid carcinoma and atypical parathyroid adenoma, and five of them were diagnosed with HPT-JT after mutational analysis. All five cases had either nonsense $C D C 73$ mutations or large $C D C 73$ deletions, which can indicate that a gross impairment of parafibromin structure can lead to parathyroid malignant transformation. However, a nonsense mutation p.Arg91* was previously described in a patient with a parathyroid adenoma (12). A gross deletion of CDC73 1-10 exons was previously described in a three-generation family with FIHP, due to adenomas, atypical adenomas and parathyroid carcinomas (26). The first description of a whole CDC73 deletion was in an 18-year-old female from HPT-JT family with a solitary parathyroid adenoma (27). In our FIHP family with the whole $C D C 73$ deletion, the proband's mother (who has not though undergone genetic testing a solitary parathyroid adenoma. Identification of one missense mutation in a patient with mild PHPT due to the necessity of routine genetic testing among all patients with PHPT under age 40 years.

There were in total 17 patients with severe osteitis fibrosa cystica associated with high total serum calcium and PTH levels and severe course of PHPT in our cohort of patients, in whom 11 had sporadic PHPT, five had HPT-JT and one had MEN1. Severe PHPT was present (O) International License. 
in clinical routine due to the development of PHPT recurrence or other endocrine tumors or the presence of a positive family history. No gross MEN1 deletions were suspected by read depth analysis of NGS data.

The pathogenicity of the identified CASR mutation has not been proven, but several in silico algorithms (Mutation Taster, SIFT-PROVEAN) predicted it to be pathogenic. Moreover, its allele frequency was not found in ExAC. We considered this mutation as likely pathogenic, but one cannot exclude that its location in C-terminal tail of the calcium-sensing receptor may not affect its function. After surgical removal of a single parathyroid adenoma, hypocalcemia and subsequent normocalcemia and normal PTH level were achieved, which is not consistent with typical FHH phenotype.

We identified mutations (one MEN1 and one CDC73) in only two FIHP families. Nevertheless, the diagnosis of FIHP in these cases can be reconsidered if other classical components of MEN1 or HPT-JT develop during the follow-up. In the remaining three families, search for novel genes responsible for FIHP development seems reasonable.

In total, hereditary forms of PHPT were identified in $1 / 3$ of patients with PHPT under 40 . In conclusion, the use of NGS seems appropriate in young PHPT patients, in particular, in those with a histological diagnosis of parathyroid carcinoma or atypical parathyroid adenoma, with suspicious positive family history, in cases of PHPT recurrence and in FIHP. Moreover, NGS is valuable in indirect assessment of large deletions, which can be further confirmed by MLPA.

\section{Declaration of interest}

The authors declare that there is no conflict of interest that could be perceived as prejudicing the impartiality of the research reported.

\section{Funding}

The study was supported by the Russian Foundation for Basic research according to the project No. 16-34-00401 мол_а.

\section{Author contribution statement}

Elizaveta Mamedova - study design, enrollment of the patients, data analysis, writing of the article. Natalya Mokrysheva - study design, enrollment of the patients, editing of the article. Evgeny Vasilyev - NGS, MLPA, data analysis. Vasily Petrov - NGS, data analysis. Ekaterina Pigarova enrollment of the patients, editing of the article. Sergey Kuznetsov surgical treatment of the patients. Nikolay Kuznetsov - surgical treatment of the patients. Liudmila Rozhinskaya - study design, editing of the article. Galina Melnichenko - study coordinator, editing of the article. Ivan Dedov - study coordinator, editing of the article. Anatoly Tiulpakov - data analysis, final editing of the article.

\section{References}

1 Silverberg SJ. Primary hyperparathyroidism. In Primer on the Metabolic Bone Diseases and Disorders of Mineral Metabolism, edn 8, ch 68, pp 543-552. Ed CJ Rosen. Ames, IA, USA: Wiley-Blackwell, 2013. (doi:10.1002/9781118453926.ch68)

2 Miller BS, Dimick J, Wainess R \& Burney RE. Age- and sex-related incidence of surgically treated primary hyperparathyroidism. World Journal of Surgery 200832 795-799. (doi:10.1007/s00268007-9427-2)

3 Alagaratnam S \& Kurzawinski TR. Aetiology, diagnosis and surgical treatment of primary hyperparathyroidism in children: new trends. Hormone Research in Paediatrics 201583 365-375. (doi:10.1159/000381622)

4 Iacobone M, Carnaille B, Palazzo FF \& Vriens M. Hereditary hyperparathyroidism - a consensus report of the European Society of Endocrine Surgeons (ESES). Langenbeck's Archives of Surgery 2015400 867-886. (doi:10.1007/s00423-015-1342-7)

5 Giusti F, Cavalli L, Cavalli T \& Brandi ML. Hereditary hyperparathyroidism syndromes. Journal of Clinical Densitometry 2013 16 69-74. (doi:10.1016/j.jocd.2012.11.003)

6 Thakker RV, Newey J, Walls GV, Bilezikian J, Dralle H, Ebeling PR, Melmed S, Sakurai A, Tonelli F \& Brandi ML. Endocrine Society clinical practice guidelines for multiple endocrine neoplasia type 1 (MEN1). Journal of Clinical Endocrinology and Metabolism 201297 2990-3011. (doi:10.1210/jc.2012-1230)

7 Jackson MA, Rich TA, Hu MI, Perrier N \& Waguespack SG. CDC73related disorders. In Gene Reviews [Internet], pp 1993-2015. Eds RA Pago, MP Adam, HH Ardinfer, et al. Seattle, WA, USA : University of Washington, 2015. (available at: http://www.ncbi.nlm.nih.gov/books/ NBK3789/)

8 Vargas-Poussou R, Mansour-Hendili L, Baron S, Bertocchio JP, Travers C, Simian C, Treard C, Baudouin V, Beltran S, Broux F, et al. Familial hypocalciuric hypercalcemia types 1 and 3 and primary hyperparathyroidism: similarities and differences. Journal of Clinical Endocrinology and Metabolism 2016101 2185-2195. (doi:10.1210/ jc.2015-3442)

9 Lassen T, Friis-Hansen L, Rasmussen ÅK, Ulrich Knigge \& FeldtRasmussen U. Primary hyperparathyroidism in young people. When should we perform genetic testing for multiple endocrine neoplasia 1 (MEN-1)? Journal of Clinical Endocrinology and Metabolism 201499 3983-3987. (doi:10.1210/jc.2013-4491)

10 Skandarajah A, Barlier N, Morlet-Barlat F, Sebag A, Enjalbert B \& Conte-Devolx JF. Henry Should routine analysis of the MEN1 gene be performed in all patients with primary hyperparathyroidism under 40 years of age? World Journal of Surgery 201034 1294-1298. (doi:10.1007/s00268-009-0388-5)

11 Langer P, Wild A, Hall A, Celik I, Rothmund M \& Bartsch DK. Prevalence of multiple endocrine neoplasia type 1 in young patients with apparently sporadic primary hyperparathyroidism or pancreatoduodenal endocrine tumours. British Journal of Surgery 2003 90 1599-1603. (doi:10.1002/bjs.4355)

12 Starker LF, Åkerström T, Long WD, Delgado-Verdugo A, Donovan P, Udelsman R, Lifton RP \& Carling T. Frequent germ-line mutations of the MEN1, CASR, and HRPT2/CDC73 genes in young patients with clinically non-familial primary hyperparathyroidism. Hormones and Cancer 20123 44-51. (doi:1007/s12672-011-0100-8)

13 Eastell R, Brandi ML, Costa AG, D'Amour P, Shoback DM \& Thakker RV. Diagnosis of asymptomatic primary hyperparathyroidism: proceedings of the fourth international workshop. Journal of Clinical Endocrinology and Metabolism 201499 3570-3579. (doi:10.1210/ jc.2014-1414)

14 Wang K, Li M \& Hakonarson H. ANNOVAR: functional annotation of genetic variants from high-throughput sequencing data. Nucleic Acids Research 201038 e164. (doi:10.1093/nar/gkq603) 
15 Plagnol V, Curtis J, Epstein M, Mok KY, Stebbings E, Grigoriadou S, Wood NW, Hambleton S, Burns SO, Thrasher AJ, et al. A robust model for read count data in exome sequencing experiments and implications for copy number variant calling. Bioinformatics 201228 2747-2754. (doi:10.1093/bioinformatics/bts526)

16 Chandrasekharappa SC, Guru SC, Manickam P, Olufemi SE, Collins FS, Emmert-Buck MR, Debelenko LV, Zhuang Z, Lubensky IA, Liotta LA, et al. Positional cloning of the gene for multiple endocrine neoplasia-type 1. Science 1997276 404-407. (doi:10.1126/ science.276.5311.404)

17 Giraud S, Zhang CX, Serova-Sinilnikova O, Wautot V, Salandre J, Buisson N, Waterlot C, Bauters C, Porchet N, Aubert JP, et al. Germline mutation analysis in patients with multiple endocrine neoplasia type 1 and related disorders. American Journal of Human Genetics 1998 63 455-467. (doi:10.1086/301953)

18 Liu W, Han X, Hu Z, Zhang X, Chen Y, Zhao Y \& Ji L. A novel germline mutation of the MEN1 gene caused multiple endocrine neoplasia type 1 in a Chinese young man and 1 year follow-up. European Review for Medical and Pharmacological Sciences 201317 3111-3116.

19 Lemmens I, Van de Ven WJM, Kas K, Zhang CX, Giraud S, Wautot V, Buisson N, De Witte K, Salandre J, Lenoir G, et al. Identification of the multiple endocrine neoplasia type 1 (MEN1) gene. The European Consortium on MEN1. Human Molecular Genetics 19976 1177-1183. (doi:10.1093/hmg/6.7.1177)

20 Bassett JH, Forbes SA, Pannett AA, Lloyd SE, Christie PT, Wooding C, Harding B, Besser GM, Edwards CR, Monson JP, et al. Characterization of mutations in patients with multiple endocrine neoplasia type 1. American Journal of Human Genetics 199862 232-244. (doi:10.1086/301729)

21 Bricaire L, Odou MF, Cardot-Bauters C, Delemer B, North MO, Salenave S, Vezzosi D, Kuhn JM, Murat A, Caron P, et al. Frequent large germline HRPT2 deletions in a French National cohort of patients with primary hyperparathyroidism. Journal of Clinical
Endocrinology and Metabolism 201398 E403-E408. (doi:10.1210/ jc.2012-2789)

22 Görtz B, Roth J, Speel EJ, Krähenmann A, De Krijger RR, Matias- Guiu X, Muletta-Feurer S, Rütmann K, Saremaslani P, Heitz PU, et al. MEN1 gene mutation analysis of sporadic adrenocortical lesions. International Journal of Cancer 199980 373-379. (doi:10.1016/S0002-9440(10)65289-3)

23 Horiuchi K, Okamoto T, Iihara M \& Tsukada T. An analysis of genotype-phenotype correlations and survival outcomes in patients with primary hyperparathyroidism caused by multiple endocrine neoplasia type 1: the experience at a single institution. Surgery Today 201343 894-899. (doi:10.1007/s00595-012-0354-y)

24 Rozhinskaya L, Pigarova E, Sabanova E, Mamedova E, Voronkova I, Krupinova J, Dzeranova L, Tiulpakov A, Gorbunova V, Orel N, et al. Diagnosis and treatment challenges of parathyroid carcinoma in a 27-year-old woman with multiple lung metastases. Endocrinology Diabetes and Metabolism Case Reports 2017 article 16-0113. (doi:10.1530/EDM-16-0113)

25 Shane E. Parathyroid carcinoma. Journal of Clinical Endocrinology and Metabolism 200186 485-493. (doi:10.1210/jcem.86.2.7207)

26 Korpi-Hyövälti E, Cranston T, Ryhänen E, Arola J, Aittomäki K, Sane T, Thakker RV \& Schalin-Jäntti C. CDC73 intragenic deletion in familial primary hyperparathyroidism associated with parathyroid carcinoma. Journal of Clinical Endocrinology and Metabolism 201499 3044-3048. (doi:10.1210/jc.2014-1481)

27 Cascón A, Huarte-Mendicoa CV, Javier Leandro-García L, Letón R, Suela J, Santana A, Costa MB, Comino-Méndez I, Landa I, Sánchez L, et al. Detection of the first gross CDC73 germline deletion in an HPT-JT syndrome family. Genes, Chromosomes and Cancer 201150 922-929. (doi:10.1002/gcc.20911)

28 Eller-Vainicher C, Chiodini I, Battista C, Viti R, Mascia ML, Massironi S, Peracchi M, D'Agruma L, Minisola S, Corbetta S, et al. Sporadic and MEN1-related primary hyperparathyroidism: differences in clinical expression and severety. Journal of Bone and Mineral Research 200924 1404-1410. (doi:10.1359/jbmr.090304)

Received in final form 4 September 2017

Accepted 4 September 2017

Accepted Preprint published online 4 September 2017 http://www.endocrineconnections.org DOI: 10.1530/EC-17-0126 (c) 2017 The authors Published by Bioscientifica Ltd

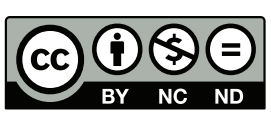

This work is licensed under a Creative Commons Attribution-NonCommercial-NoDerivs 4.0 International License. 